\title{
Analysis of the complex interaction of CDR1as-miRNA-protein and detection of its novel role in melanoma
}

\author{
LIHUAN ZHANG ${ }^{*}$, YUAN LI ${ }^{*}$, WENYAN LIU, HUIFENG LI and ZHIWEI ZHU \\ College of Life Sciences, Shanxi Agricultural University, Taigu, Shanxi 030801, P.R. China
}

Received May 15, 2017; Accepted April 9, 2018

DOI: $10.3892 / \mathrm{ol} .2018 .8700$

\begin{abstract}
Despite improvements in the prevention, diagnosis and treatment of melanoma having developed rapidly, the role of circular RNA CDR1 antisense RNA (CDR1as) in melanoma remains to be elucidated. The aim of the present study was to predict the novel roles of CDR1as in melanoma through novel bioinformatics analysis. In the present study, the circ2Traits database was used to supply information on CDRlas in cancer. CircNet, circBase and circInteractome databases were used to detect the co-expression of CDR1as, microRNAs and proteins. Furthermore, the functions and pathways of the associated proteins were predicted using the Database for Annotation, Visualization and Integrated Discovery. Gene Ontology enrichment analysis suggested that the proteins associated with CDRlas were mainly regulated in the cytoplasm as the molecular protein binding, the biological process of cell division and the endoplasmic reticulum tubular network, which are all involved in cancer-related processes. The Kyoto Encyclopedia of Genes and Genome pathway cell cycle was closely associated with the location of cancer. Co-expression network analysis revealed the associations among CDRlas, microRNAs and proteins. The bioinformatics analysis showed that CDRlas may act as the competing endogenous RNA for the vital genes, which are associated with the invasion and migration of melanoma.
\end{abstract}

\section{Introduction}

Circular RNA (circRNA) was first detected in RNA viruses in the 1970s (1,2), in which the $3^{\prime}$ and $5^{\prime}$ ends are joined together. Compared with the linear structure of coding RNAs, the closed structure of circRNA means it is more conserved (3). CircRNA

Correspondence to: Ms. Lihuan Zhang, College of Life Sciences, Shanxi Agricultural University, 1 Mingxiannan Road, Taigu, Shanxi 030801, P.R. China

E-mail: 18234475242@163.com

*Contributed equally

Key words: interaction, circular RNA CDR1 antisense RNA, microRNA, role, melanoma has been detected in several species, including viruses (2), plants (4), archaea (5)[Salzman, 2013 \#198; Memczak, 2013 \#291] and animals (6). In eukaryotic cells, circRNA has attracted attention due to its unique characteristics, including high stability, specificity and evolutionary conservation (7). The majority of circRNAs are derived from exons of coding regions, 3'UTRs, 5'UTRs, introns, intergenetic regions and antisense RNAs (8). CircRNAs, which have attracted attention in recent years, can be produced by canonical and noncanonical splicing as distinguished from the linear RNAs (9). Through high-throughput sequencing, three types of circRNAs have been identified: Exonic circRNAs (7), circular intronic RNAs (ciRNAs) (9), and retained-intron circular RNAs or exon-intron circRNAs (elciRNAs) (10). To date, evidence suggests that circRNAs may regulate transcription and pathways by manipulating microRNAs $(11,12)$. circRNA_100290 acts as a competing endogenous RNA to regulate the expression of cyclin-dependent kinase (CDK)6 through regulating microRNA (miR)-29b family members (13). circRNA-100269 and miR-630 comprise a novel pathway, which regulates the proliferation of gastric cancer cells (14). Circ-ABCB10 has provided novel insight into the pathogenesis of breast cancer through regulating miR-1271 (15). Therefore, circRNAs are involved in the development of diseases and have become novel biomarkers in cancer $(16,17)$.

CDRlas, as a circRNA with the most known miR-7 binding sites ( $>70)$, is known for its 'circular RNA sponge for miR-7' (ciRS-7 and CDR1as), and there are $>70 \mathrm{miR}-7$ binding sites on this circRNA $(3,18)$. CDRlas, as an endogenous RNA, indirectly regulates microRNAs and is involved in the development of multiple diseases through a variety of pathways $(18,19)$. A previous study showed that the CDR1as-miR-7 axis can regulate the neocortical and hippocampal neurons in mouse brains $(17,20)$. In the embryonic zebrafish midbrain, the overexpression of CDR1as induces developmental defects (6). These findings indicate that CDRlas is important in different mechanisms, acting as an miR-7 buffer $(18,21)$. Other functional investigations have shown that ciRS-7 inhibits the activity of miR-7 and activates downstream genes of miR-7 to perform a regulatory role in various types of cancer, including colorectal cancer (17), hepatocellular carcinoma (16) and glioblastoma multiforme (22).

Melanoma, the most life-threatening form of skin cancer, has changed markedly in the 21 st century. Early detection, genetic testing and substantial improvements in advanced melanoma 
therapies are examples of such progress. However, the role of circRNA in melanoma remains to be fully elucidated. miR-7 can target melanoma through inhibiting the mitogen-activated protein kinase (MAPK) and phosphoinositide 3-kinase (PI3K)/protein kinase B (AKT) signaling pathways (23), therefore, CDR1as serves as a novel and stable biomarker for the diagnosis and progression of several types of cancer, including melanoma, which is the most difficult form of skin cancer to treat (24).

In the present study, the unknown roles of CDR1as in a highly complex metabolic network were examined through novel bioinformatics analysis as a novel field of investigation to elucidate its full biologic functions in cellular regulation and in human diseases.

\section{Materials and methods}

Bioinformatics databases and software. Several databases and software packages were used in the present study. The University of California, Santa Cruz (UCSC) Genome Browser (http://genome.UCSC.Edu/), TargetScan (25) (http://www. Targetscan.Org/vert_61) predicts biological targets of miRNAs by searching for the presence of conserved $8 \mathrm{mer}, 7 \mathrm{mer}$ and 6 mer sites, which match the seed region of each miRNA (26). In mammals, predictions are ranked based on the predicted efficacy of targeting as calculated using cumulative weighted context++ scores of the sites (27).

The Database for Annotation, Visualization and Integrated Discovery (DAVID; http://david.abcc. Nciferf. gov/) $(28,29)$ involves the Statistical Consultation Group, Computer \& Statistical Services Program, NCI-Frederick and NIH (30).

CircBase (http://www.circbase.org/) (31) contains data from all studies of large-scale circRNA identification published to date. In addition to the information made available by the investigators, the present study annotated all circRNA transcripts and predicted their putative spliced forms. Transcript reannotation was necessary to standardize the content and level the quantity of information contained across certain publications $(10,32)$.

For CircNet (http://circnet.mbc.nctu.edu.tw/) (33), perfect complementarity was required of sequences for a circRNA-miRNA target association to be identified. To normalize the number of occurrences of these sites, the following formula was used: Frequency of Nmer=(number of target seeds $x 1,000) /(\mathrm{N} \times$ length of circRNA). With this formula, four frequency numbers can be acquired from each circRNA-miRNA pair. To distinguish circRNA from linear isoforms, frequency values were also calculated for linear mRNA and miRNA pairs. P-values for each circRNA and miRNA association were acquired by one minus the calculated cumulated distribution function of the frequency $\mathrm{Z}$ score. For a circRNA-miRNA pair, $\mathrm{P}<0.001$ indicates high regulatory potential between the circRNA and miRNA (33).

CircInteractome (http://circinteractome.nia.nih.gov/) comprises datasets, including the binding sites of $35 \mathrm{RBPs}$ identified using PAR-CLIP, HITS-CLIP, or iCLIP, retrieved from starBase v2.0 (http://starbase.sysu.edu.cn/), which decodes miRNA-ceRNA, miRNA-ncRNA and protein-RNA interaction networks from large-scale CLIP-Seq data (34).
Using Circ2Traits (http://gyanxet-beta.com/circdb/), a measure was implemented for the likelihood of a circRNA to be associated with a disease in terms of the significance of the interaction between the circRNA and miRNAs associated with the disease of interest. In the present study, P-values were calculated for the association between each circRNA interacting with disease-associated miRNAs. The $\mathrm{p}$-value for the likelihood of the circRNA to be enriched for interaction with miRNAs associated with that disease was calculated as follows: $\mathrm{P}=\sum \mathrm{i}=\mathrm{mdmin}$ (mc,Md) (Mdi) (MT-Mdmc-i) (MTmc). MT is the total number of miRNAs in the human genome, mc is the number of miRNAs interacting with the circRNA, Md is the total number of miRNAs associated with the disease, md is the number of miRNAs associated with the disease which interact with the circRNA. Following Bonferroni correction, $\mathrm{P}<0.05$ indicated the circRNA was likely to be associated with the disease, and pthreshold $=0.05 / \mathrm{mm}$ indicated the total number of circRNAs interacting with any miRNA associated with the disease (35).

MiRanda (http://.Mirorna.Org/microrna/getDownload) is an mirSVR predicted target site scoring method: Comprehensive modeling of microRNA targets predicts functional non-conserved and non-canonical sites (mirSVRscore <-1) (36).

Methods of analysis. The location of CDR1as in human and mouse genomes were supplied by the UCSC Genome Browser database. The roles of CDR1as in cancer are summarized in the circ2Traits database. The CircBase, circNet, miRanda, TargetScan and circInteractome databases were used to detect the interaction and co-expression of CDR1as, miRNAs and proteins. The functions and the Kyoto Encyclopedia of Genes and Genomes (KEGG) pathways of the associated proteins were predicted using DAVID.

\section{Results}

Location of CDRlas. CDRlas is derived from an antisense transcript of the CDR1 at chromosome X (NC_004065) in humans and chromosome X (NC_000086.7) in mice, located in the ChrX:139865339-139866824 and chX:58436422-58539349 (Fig. 1).

MicroRNA prediction. Correlations between circRNAs and microRNAs were determined using CircInteractome and TargetScan. CDR 1as contained $>70$ conserved sites for miR-7, in addition to miR-671-5p, miR-3134 and miR-139-3p, which are important target miRNAs regulating metabolism. The predicted microRNAs in these two databases comprised 15 combined microRNAs, as shown in the Table I (context score percentile $>90$ ).

Protein prediction. The predicted proteins were all targeted by the microRNAs interacting with CDR1as. A total of 60 proteins were identified using the cirNet database and miRanda database in total $(\mathrm{P}<0.05$, mirSVRscore $<-1)$. Several of these proteins are associated with cancer invasion and metastasis, including AKT3, Secretory carrier-associated membrane protein 2 (SCAMP2), SLU7, B-cell lymphoma 2 (BCL2) and CDK1. BCL2 is an important gene, which inhibits the apoptotic death of certain cells, including lymphocytes. 
Table I. miRs interacting with CDR1as.

Circular RNA
$\operatorname{miR}$

CDR1as

miR-7-5p, miR-671-5p, miR-3134, miR-139-3p, miR-6807-5p, miR-3617-5p, miR-3617-3p, miR-4254, miR-3145-3p, miR-4291, miR-3201, miR-4306, miR-471-5p, miR-3065-3p, miR-3132,

miRs were at the intersection of the CircInteractome and TargetScan databases. CDR1as, circular RNA CDR1 antisense RNA; miR, miroRNA.

Table II. Target proteins of microRNAs interacting with CDR1as.

\begin{tabular}{ll} 
Circular RNA & \multicolumn{1}{c}{ MicroRNA-interacted protein } \\
\hline CDR1as & PAPD7, PTN3, CDKIAP2, C11orf74, PPIF, TUBA1B, CDR1, ABHD14A-ACY1, GNA13, AKT3, GJC1, \\
& SCAMP2, CNE3, MED16, WASF2, LRPPRC, URGCP-MRPS24, OCID2, OCIAD2, SNRNP27, ZNF730, \\
& PARP2, TMEM170A, CHEK1, SMC4, MGAT4A, TRAF3IP2, ZNF689, SCUBE3, FAM13A, MTRNRIL3, \\
& RAB10, Bc1212-PABPN1, ARIH2, CYB5D1, ZBTB33, FEM1B, GNA13, CDK6, MTHFD2, IVNS1ABP, \\
& NUPL2, ARPP19, C17orf49, PNRC1, RNF103-CHMP3, USF, MICA, SLU7, BTBD9, MYL12B, CDKN2B, \\
& C1QTNF6, DCAF7, NBPF10, STX1B, ABCC5, SNX20, VAS1V
\end{tabular}

Target proteins identified at the intersection of the CircNet database and MiRanda database prediction.

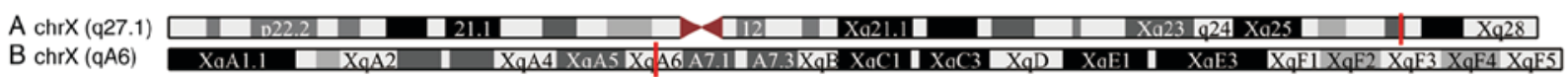

Figure 1. Location of CDR1as. The red lines indicate the location of CDR1 as in chromosome. The location of the CDR1as was provided by University of California, Santa Cruz genome browser. (A) Human genome; (B) mouse genome. CDR1as in these two species was located in chromosome X. CDR1as, circular RNA CDR1 antisense RNA.

CDK1 functions as a serine/threonine kinase, and is key in cell cycle regulation (Table II).

circRNA-microRNA-protein co-expression network. The potential microRNA-circRNA-mRNA interaction analysis, which was determined using the circNet database, was used to elucidate the correlations between circRNAs and microRNA. In the present study, the top 15 miRNAs that interacted with CDR1as were selected (yellow in Fig. 2), and 66 mRNAs (light green in Fig. 2), were identified as the targets of these miRNAs $(\mathrm{P}<0.001$ and support experiment $>5)$.

Gene Ontology (GO) and KEGG) pathway analysis of target genes. The results of the GO analysis revealed target genes $(\mathrm{P}<0.05$, Bonferroni corr $<0.05)$ involved in biological processes, including cell division and $\mathrm{G} 2 / \mathrm{M}$ transition, which can negatively regulate the intrinsic apoptotic signaling pathway in the mitotic cell cycle. The main molecular function of these target genes was protein binding. The cellular components of the target genes comprised nucleoplasms, cytoplasm and condensed nuclear chromosomes (Fig. 3A). These processes are all associated with human tumorigenesis and cancer metastasis. The results of the KEGG analysis showed three pathways $(\mathrm{P}<0.05)$ associated with the target genes; these pathways were small cell lung cancer, cell cycle and platelet activation (Fig. 3B).

CDRlas and cancer. CDRlas was found to be involved in several types of cancer, including gastric cancer, hepatic

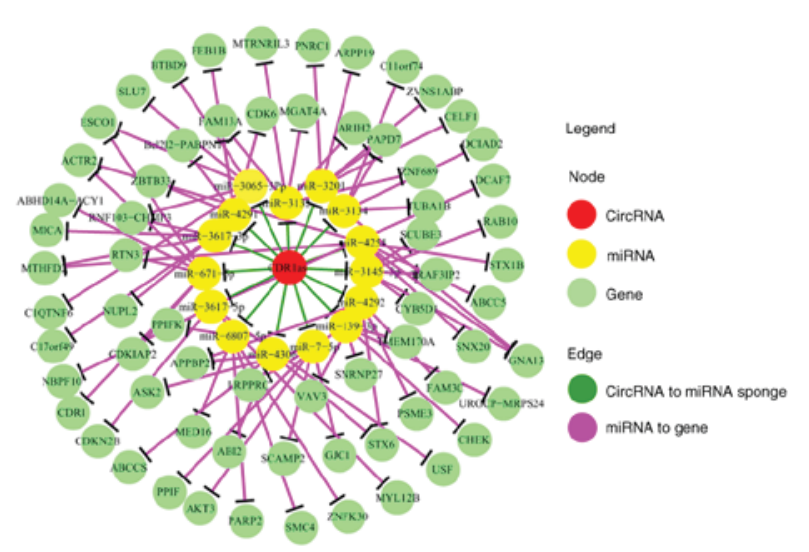

Figure 2. CDR1as-miRNA-protein co-expression regulatory network. The network was analyzed in the CircNet database. circRNA, circular RNA; miR/miRNA, microRNA; CDR1as, circular RNA CDR1 antisense RNA.

carcinoma, lung cancer, breast cancer, glioma, diabetes, Parkinson's disease, cervical cancer and melanoma. These findings were predicted in the Circ2Traits database, which also supplies the associated protein. BCL-2 is regulated by the CDR1as/miR-7 axis, which is involved in Parkinson's disease and lung cancer. Phosphatidylinositol 3-kinase catalytic subunit $\delta$ is associated with hepatic carcinoma. p21-activated kinase-1 (PAK1) and Kruppel-like factor 4 (KLF4) are important in breast cancer. Insulin receptor 

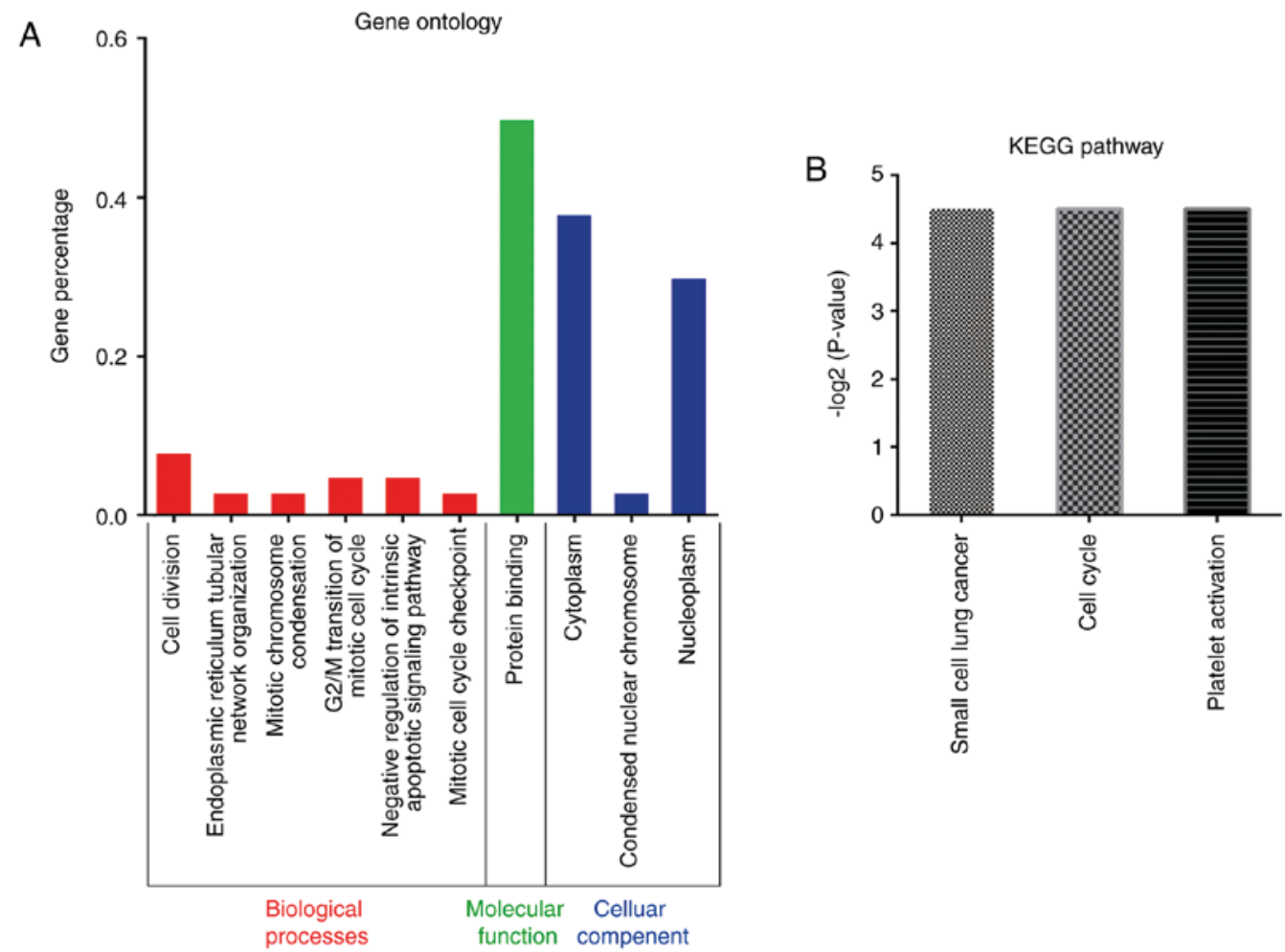

Figure 3. GO enrichment and KEGG pathway enrichment. (A) GO enrichment. The red column represents the biological process; the green column represents the molecular function; the blue column represents the cellular component. The Y-axis represents the gene percentage; the X-axis represents the GO terms $(\mathrm{P}<0.05$, Benjamini <0.05). (B) KEGG pathway enrichment. GO, Gene Ontology; KEGG, Kyoto Encyclopedia of Genes and Genomes.

substrate (IRS)-2, IRS-1 and PAK are associated with glioma. IRS-2, epidermal growth factor receptor (EGFR), IGE-1, CRAF and paired box 6 (PAX6) have all been reported be vital in the melanoma through interacting with miR-7, therefore, CDR1as may offer novel clues for the treatment of melanoma. The predictions of CDR1as-miR-7-mRNAs in different types of cancer are shown in. Fig. 4. These mRNAs associated with cancer have been reported and are the targets of miR-7, therefore, CDR1as may be important in regulating these types of cancer.

\section{Discussion}

With the development of novel biochemical and computational approaches in the RNA field, circRNAs have been an active area of investigation (37). CircRNAs, unlike linear RNAs, form a covalently closed continuous loop (38); circRNAs are also resistant to RNase R treatment (39). CircRNAs interact with other molecules or microRNAs to regulate gene expression in metabolism at the post-transcriptional or transcriptional level (40). CircRNAs are also crucial in various biological processes associated with cancer cell development and proliferation, in addition to cancer metastasis and invasion $(3,19)$.

CDR1as, which is also considered a circRNA sponge for miR-7 (ciRS-7), is derived from the antisense of cerebellar degeneration-related protein 1 transcript (40), and has attracted attention in the RNA field. CDR1as has numerous pathways and diseases of note, including its function as a ceRNA of microRNA, particularly miR-7, in several types of cancer. miR-7 can bind in the 3' UTRs of mRNAs, which is vital in

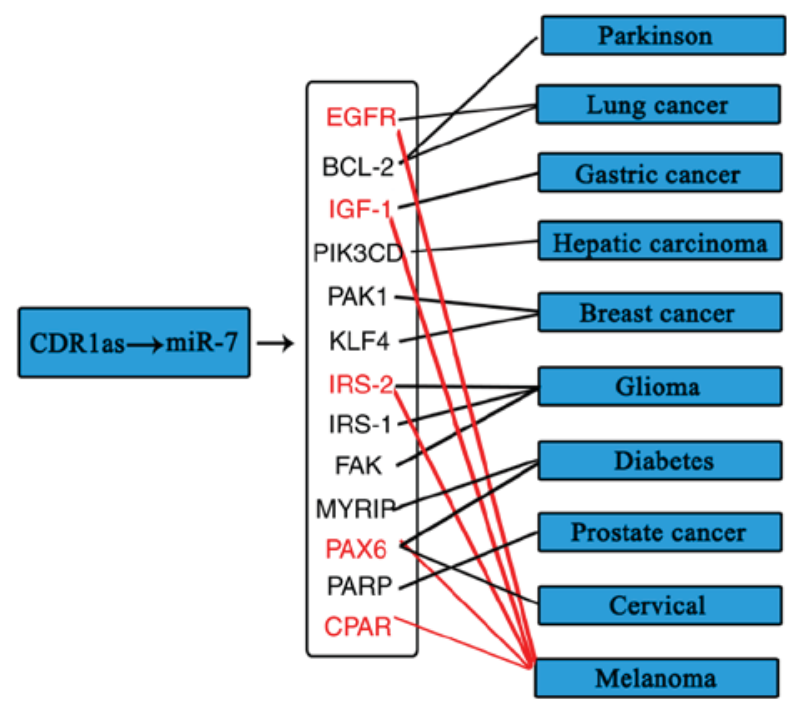

Figure 4. CDR1as/miR-7 and cancer. CDR1as/miR-7 was found to regulate the expression of several cytokines and oncogenes in different types of cancer. The red names and lines indicate the genes and relationship with melanoma. The black names and lines indicate the genes and relationship with other cancers. miR, microRNA; CDR1as, circular RNA CDR1 antisense RNA; EGFR, epidermal growth factor receptor; BCL-2, B cell lymphoma-2; IGF-1, insulin-like growth factor-1; PIK3CD, phosphatidylinositol 3-kinase catalytic subunit $\delta$; PAK, p21-activated kinase; KLF4, Kruppel-like factor 4; IRS, insulin receptor substrate; FAK, focal adhesion kinase; MYRIP, myosin VIIA and Rab interacting protein; PARP, poly (ADP-ribose) polymerase; CPAR, histone H3-like centromeric protein cpar-1.

these types of cancer, including breast cancer (41), glioma (42), melanoma (23), diabetes (43), Parkinson's disease (44) and 
cervical cancer (45). As CDRlas/miR-7 is likely to be involved in various types of cancer, including breast cancer, glioma, melanoma, diabetes, Parkinson's disease and cervical cancer, detecting the function of CDR1as in the initiation and progression of cancer is of interest for further investigations (9). CDRlas is a common genetic trigger in human diseases and a specific combination of various markers for early diagnosis in cancer.

In the present study, GO enrichment analysis revealed that the miR-7 target genes are associated with several crucial biological processes and are involved in the cellular response during cancer development. Among the pathways identified in the present study, platelet activation has been shown to be a main regulator in the tumor cell response (46). Negative regulation of the intrinsic apoptotic signaling pathway can increase the repair space of DNA double-strand breaks (47). miR-7 can markedly decrease the expression levels of EGFR, IGF-1R and CRAF, and suppress the activation of MAPK and the PI3K/AKT pathway in VemR A375 melanoma cells (23). IRS-2 is a target of miR-7, and promotes migration and Akt signaling in melanoma (24). Pax6 is associated with the Wnt signaling pathway, which regulates the invasion and migration of glioblastoma (48), prostate cancer (49) and bladder cancer (50). Pax6 is also important in the growth of malignant melanoma (51). As Pax6 is the target of miR-7 (52), the CDRlas-miR-7-Pax6 axis may be important in cancer invasion and migration.

Melanoma is a the main type of refractory skin cancer due to its poor prognosis and high chronicity (53). The CDR1as/miR-7 axis is likely to be involved in several types of cancer, including melanoma (54). Additionally, IRS-2 is a target of miR-7-5p in melanoma cells and activates Akt, which promotes melanoma cell migration. The inhibition of IRS-2 reduces the activity of Akt, a key oncogenic effector molecule in melanoma, and this finding suggests that the co-interaction of IRS-2 and CDR1as is a therapeutic target in melanoma, with the potential function to inhibit PI3K/Akt signaling, cell migration and tumor metastasis (55).

The genome-wide identification of circRNAs depends on efficient bioinformatics analysis. The prediction and functional analysis of target genes are under strict conditions, which can effectively reduce the false prediction rate. However, there can be errors in the screening rates of the analysis, and a more accurate method for subsequent analysis and further assessment is required. Several results have been collected from several studies, including the interaction of CDR1as and miR-7 (56), and the interaction of miR-7 and proteins $(23,57)$, however, there has been no systematic investigation of the CDR1as-miRNA-protein axis in melanoma or several other types of cancer. The present study aimed to offer guidelines for further investigations of the CDR1as-miRNA-protein axis in cancer.

In conclusion, decades have passed since circRNAs were first detected. CircRNAs have attracted increasing attention due to improvements in high-throughput sequencing technologies and bioinformatics progression. In the present study, GO enrichment analysis suggested that the protein associated with CDR1as is mainly regulated in the cytoplasm, as the molecular protein binding, the biological process of cell division, and the endoplasmic reticulum tubular network may be involved in cancer. The KEGG pathway enrichment cell cycle is closely associated with the treatment of cancer. Co-expression network analysis revealed the association between CDR1as, miR-7 and proteins; and CDR1as was been found to be important in several types of cancer, including breast cancer, glioma, diabetes, Parkinson's disease, and melanoma in particular. These results are likely to assist in future investigations of the function of CDR1as as a novel therapeutic target in cancer.

\section{Acknowledgements}

The authors would like to thank Biomarker Technologies, Inc. (Beijing, China) for technical support.

\section{Funding}

This study was supported by a grant from the Science and Technology of Shanxi Agricultural University, Shanxi, China (grant no. 2016ZZ07) and the National Natural Science Foundation of China (grant no. 31402156).

\section{Availability of data and materials}

The datasets used and/or analyzed during the present study are available from the corresponding author on request.

\section{Authors' contributions}

LZ and YL performed most of the research and were major contributors towards writing the manuscript. WL and HL collected and analyzed the data. ZZ and LZ made substantial contributions to the design of the work, drafted the manuscript and revised it critically for important intellectual content. LZ and YL gave final approval of the version to be published.

\section{Ethics approval and consent to participate}

Not applicable

\section{Consent for publication}

Not applicable.

\section{Competing interests}

The authors declare that they have no competing interests.

\section{References}

1. Kos A, Dijkema R, Arnberg AC, van der Meide PH and Schellekens H: The hepatitis delta (delta) virus possesses a circular RNA. Nature 323: 558-560, 1986.

2. Sanger HL, Klotz G, Riesner D, Gross HJ and Kleinschmidt AK: Viroids are single-stranded covalently closed circular RNA molecules existing as highly base-paired rod-like structures. Proc Natl Acad Sci USA 73: 3852-3856, 1976.

3. Guo JU, Agarwal V, Guo H and Bartel DP: Expanded identification and characterization of mammalian circular RNAs. Genome Biol 15: 409, 2014.

4. Lu T, Cui L, Zhou Y Zhu C, Fan D, Gong H, Zhao Q, Zhou C, Zhao Y, Lu D, et al: Transcriptome-wide investigation of circular RNAs in rice. RNA 21: 2076-2087, 2015. 
5. Salzman J, Gawad C, Wang PL, Lacayo N and Brown PO Circular RNAs are the predominant transcript isoform from hundreds of human genes in diverse cell types. PLoS One 7: e30733, 2012.

6. Memczak S, Jens M, Elefsinioti A, Torti F, Krueger J, Rybak A, Maier L, Mackowiak SD, Gregersen LH, Munschauer M, et al: Circular RNAs are a large class of animal RNAs with regulatory potency. Nature 495: 333-338, 2013.

7. Jeck WR, Sorrentino JA, Wang K, Slevin MK, Burd CE, Liu J, Marzluff WF and Sharpless NE: Circular RNAs are abundant, conserved, and associated with ALU repeats. RNA 19: 141-157, 2013.

8. Li Y, Zheng Q, Bao C, Li S, Guo W, Zhao J, Chen D, Gu J, He X and Huang S: Circular RNA is enriched and stable in exosomes: A promising biomarker for cancer diagnosis. Cell Res 25: 981-984, 2015

9. Zhang XO, Dong R, Zhang Y, Zhang JL, Luo Z, Zhang J, Chen LL and Yang L: Diverse alternative back-splicing and alternative splicing landscape of circular RNAs. Genome Res 26: 1277-1287, 2016.

10. Salzman J, Chen RE, Olsen MN, Wang PL and Brown PO Cell-type specific features of circular RNA expression. PLoS Genet 9: e1003777, 2013.

11. Zhao ZJ and Shen J: Circular RNA participates in the carcinogenesis and the malignant behavior of cancer. RNA Biol 14 514-521, 2017.

12. Jeck WR and Sharpless NE: Detecting and characterizing circular RNAs. Nat Biotechnol 32: 453-461, 2014.

13. Chen L, Zhang S, Wu J, Cui J, Zhong L, Zeng L and Ge S circRNA_100290 plays a role in oral cancer by functioning as a sponge of the miR-29 family. Oncogene 36: 4551-4561, 2017.

14. Yan Z, Hao L, Li W, Yu J, Li J, Shen Z, Ye G, Qi X and Li G: CircRNA_100269 is downregulated in gastric cancer and suppresses tumor cell growth by targeting miR-630. Aging (Albany NY) 9: 1585-1594, 2017.

15. Liang HF, Zhang XZ, Liu BG, Jia GT and Li WL: Circular RNA circ-ABCB10 promotes breast cancer proliferation and progression through sponging miR-1271. Am J Cancer Res 7: 1566-1576, 2017.

16. Xu L, Ming Z, Zheng X, Yi P, Lan $\mathrm{C}$ and $\mathrm{Xu}$ M: The circular RNA ciRS-7 (Cdrlas) acts as a risk factor of hepatic microvascular invasion in hepatocellular carcinoma. J Cancer Res Clin Oncol 143: 17-27, 2017.

17. Tang W, Ji M, He G, Yang L, Niu Z, Jian M, Wei Y, Ren L and $\mathrm{Xu}$ J: Silencing CDR1as inhibits colorectal cancer progression through regulating microRNA-7. Onco Targets Ther 10 2045-2056, 2017.

18. Xu H, Guo S, Li W and Yu P: The circular RNA Cdrlas, via miR-7 and its targets, regulates insulin transcription and secretion in islet cells. Sci Rep 5: 12453, 2015.

19. Hansen TB, Jensen TI, Clausen BH, Bramsen JB, Finsen B, Damgaard CK and Kjems J: Natural RNA circles function as efficient microRNA sponges. Nature 495: 384-388, 2013.

20. Hansen TB, Wiklund ED, Bramsen JB, Villadsen SB, Statham AL, Clark SJ and Kjems J: miRNA-dependent gene silencing involving Ago2-mediated cleavage of a circular antisense RNA. EMBO J 30: 4414-4422, 2011

21. Hansen TB, Kjems J and Damgaard CK: Circular RNA and miR-7 in cancer. Cancer Res 73: 5609-5612, 2013.

22. Barbagallo D, Condorelli A, Ragusa M, Salito L, Sammito M, BanelliB,CaltabianoR,BarbagalloG,ZappalàA,BattagliaR,etal: Dysregulated miR-671-5p/CDR1-AS/CDR1/VSNL1 axis is involved in glioblastoma multiforme. Oncotarget 7: 4746-4759, 2016.

23. Sun X, Li J, Sun Y, Dong L, Shen C, Yang L, Yang M, Li Y, Shen G, Tu Y and Tao J: miR-7 reverses the resistance to BRAFi in melanoma by targeting EGFR/IGF-1R/CRAF and inhibiting the MAPK and PI3K/AKT signaling pathways. Oncotarget 7 : 53558-53570, 2016.

24. Giles KM, Brown RA, Epis MR, Kalinowski FC and Leedman PJ: miRNA-7-5p inhibits melanoma cell migration and invasion. Biochem Biophys Res Commun 430: 706-710, 2013

25. Jr PA, Hood N, Lyn S, Foote J and Bennett J: MP-4.09: TargetScan ${ }^{\circledR}$ : A novel approach for outpatient prostate biopsy with the potential for use as an aid to focal prostate therapy. Urology 72: S86-S87, 2008.

26. Lewis BP, Burge CB and Bartel DP: Conserved seed pairing, often flanked by adenosines, indicates that thousands of human genes are microRNA targets. Cell 120: 15-20, 2005.
27. Agarwal V, Bell GW, Nam JW and Bartel DP: Predicting effective microRNA target sites in mammalian mRNAs. Elife 4: e05005, 2015

28. Dennis G Jr, Sherman BT, Hosack DA, Yang J, Gao W, Lane HC and Lempicki RA: DAVID: database for annotation, visualization, and integrated discovery. Genome Biol 4: P3, 2003.

29. Huang da W, Sherman BT and Lempicki RA: Systematic and integrative analysis of large gene lists using DAVID bioinformatics resources. Nat Protoc 4: 44-57, 2009.

30. Huang DW, Sherman BT, Tan Q, Collins JR, Alvord WG, Roayaei J, Stephens R, Baseler MW, Lane HC and Lempicki RA: The DAVID gene functional classification tool: A novel biological module-centric algorithm to functionally analyze large gene lists. Genome Biol 8: R183, 2007.

31. Glažar P, Papavasileiou P and Rajewsky N: circBase: A database for circular RNAs. RNA 20: 1666-1670, 2014

32. Zhang Y, Zhang XO, Chen T, Xiang JF, Yin QF, Xing YH, Zhu S, Yang L and Chen LL: Circular intronic long noncoding RNAs. Mol Cell 51: 792-806, 2013.

33. Liu YC, Li JR, Sun CH, Andrews E, Chao RF, Lin FM, Weng SL, Hsu SD, Huang CC, Cheng C, et al: CircNet: A database of circular RNAs derived from transcriptome sequencing data. Nucleic Acids Res 44: D209-D215, 2016.

34. Dudekula DB, Panda AC, Grammatikakis I, De S, Abdelmohsen K and Gorospe M: CircInteractome: A web tool for exploring circular RNAs and their interacting proteins and microRNAs. RNA Biol 13: 34-42, 2016.

35. Ghosal S, Das S, Sen R, Basak P and Chakrabarti J: Circ2Traits: A comprehensive database for circular RNA potentially associated with disease and traits. Front Genet 4: 283, 2013.

36. John B, Sander C and Marks DS: Prediction of human miRNA targets. Methods Mol Biol 342: 101-113, 2006.

37. Ebbesen KK, Kjems J and Hansen TB: Circular RNAs: Identification, biogenesis and function. Biochim Biophys Acta 1859: 163-168, 2016

38. Lasda E and Parker R: Circular RNAs: Diversity of form and function. RNA 20: 1829-1842, 2014.

39. Chen I, Chen CY and Chuang TJ: Biogenesis, identification, and function of exonic circular RNAs. Wiley Interdiscip Rev RNA 6: 563-579, 2015.

40. Zhang Y, Sun L, Xuan L, Pan Z, Li K, Liu S, Huang Y, Zhao X, Huang L, Wang Z, et al: Reciprocal changes of circulating long non-coding RNAs ZFAS1 and CDR1AS predict acute myocardial infarction. Sci Rep 6: 22384, 2016.

41. Raychaudhuri M, Bronger H, Buchner T, Kiechle M, Weichert W and Avril S: MicroRNAs miR-7 and miR-340 predict response to neoadjuvant chemotherapy in breast cancer. Breast Cancer Res Treat 162: 511-521, 2017.

42. Liu LH, Zhang HY, Liu XZ, Zhang LD, Jiang ZM and Hospital TH: Correlation between miR-7 and expression of EGFR/PI3K signal pathway related protein in glioma. Shandong Med J, 2014.

43. Wan S, Wang J, Wang J, Wu J, Song J, Zhang CY, Zhang C, Wang C and Wang JJ: Increased serum miR-7 is a promising biomarker for type 2 diabetes mellitus and its microvascular complications. Diabetes Res Clin Pract 130: 171-179, 2017.

44. Li S, Lv X, Zhai K, Xu R, Zhang Y, Zhao S, Qin X, Yin L and Lou J: MicroRNA-7 inhibits neuronal apoptosis in a cellular Parkinson's disease model by targeting Bax and Sirt2. Am J Transl Res 8: 993-1004, 2016.

45. Liu S, Zhang P, Chen Z, Liu M, Li X and Tang H: MicroRNA-7 downregulates XIAP expression to suppress cell growth and promote apoptosis in cervical cancer cells. FEBS Lett 587: 2247-2253, 2013

46. Toulany M and Rodemann HP: Phosphatidylinositol 3-kinase/Akt signaling as a key mediator of tumor cell responsiveness to radiation. Semin Cancer Biol 35: 180-190, 2015.

47. Wang PL, Bao Y, Yee MC, Barrett SP, Hogan GJ, Olsen MN, Dinneny JR, Brown PO and Salzman J: Circular RNA is expressed across the eukaryotic tree of life. PLoS One 9: e90859, 2014.

48. Kim Y, Kim KH, Lee J, Lee YA, Kim M, Lee SJ, Park K, Yang H, Jin J, Joo KM, et al: Wnt activation is implicated in glioblastoma radioresistance. Lab Invest 92: 466-473, 2012.

49. Cojoc M, Peitzsch C, Kurth I, Trautmann F, Kunz-Schughart LA, Telegeev GD, Stakhovsky EA, Walker JR, Simin K, Lyle S, et al: Aldehyde dehydrogenase is regulated by $\beta$-Catenin/TCF and promotes radioresistance in prostate cancer progenitor cells. Cancer Res 75: 1482-1494, 2015.

50. Renaud Pierre Q: MIR-7 targets pax6 and modulates bladder cancer cell migration, Feb 2, 2017. 
51. Treszl A, Ladanyi A, Rakosy Z, Buczko Z, Adany R and Balazs M: Molecular cytogenetic characterization of a novel cell line established from a superficial spreading melanoma. Front Biosci 11: 1844-1853, 2006

52. Needhamsen M, White RB, Giles KM, Dunlop SA and Thomas MG: Regulation of Human PAX6 Expression by miR-7. Evol Bioinform Online 10: 107-113, 2014.

53. Bonazzi VF, Stark MS and Hayward NK: MicroRNA regulation of melanoma progression. Melanoma Res 22: 101-113, 2012.

54. Geng HH, Li R, Su YM, Xiao J, Pan M, Cai XX and Ji XP: The Circular RNA Cdrlas promotes myocardial infarction by mediating the regulation of miR-7a on its target genes expression. PLoS One 11: e0151753, 2016.
55. Fang Y, Xue JL, Shen Q, Chen J and Tian L: MicroRNA-7 inhibits tumor growth and metastasis by targeting the phosphoinositide 3-kinase/Akt pathway in hepatocellular carcinoma. Hepatology 55: 1852-1862, 2012.

56. Yu L, Gong X, Sun L, Zhou Q, Lu B and Zhu L: The circular RNA cdrlas act as an oncogene in hepatocellular carcinoma through targeting miR-7 expression. PLoS One 11: e0158347, 2016.

57. Shen Z, Qin X, Yan M, Li R, Chen G, Zhang J and Chen W: Cancer-associated fibroblasts promote cancer cell growth through a miR-7-RASSF2-PAR-4 axis in the tumor microenvironment. Oncotarget 8: 1290-1303, 2017. 